\title{
A Singular Point in the Characteristics of the Serial RLC Circuit
}

\author{
Parfentiev Nikolay Andreevich ${ }^{1}$, Parfentieva Natalia Andreevna ${ }^{2}$ \\ ${ }^{1}$ All-Russian Research Institute for Optophysical Measurements (VNIIOFI), Moscow, Russia \\ ${ }^{2}$ Department of Physics, Moscow State University of Civil Engineering, Moscow, Russia
}

Email address:

nik_parfenium@mail.ru (P. N. Andreevich)

To cite this article:

Parfentiev Nikolay Andreevich, Parfentieva Natalia Andreevna. A singular point in the characteristics of the serial RLC circuit. American Journal of Physics and Applications. Vol. 5, No. 6, 2017, pp. 109-112. doi: 10.11648/j.ajpa.20170506.17

Received: August 18, 2017; Accepted: September 4, 2017; Published: November 8, 2017

\begin{abstract}
It is shown that the frequency characteristics of the RCL serial circuit have a special point at which the modulus of the complex resistance is not dependent on resistance. Is investigated the existence region of the real model of the serial circuit. A comparison of different schemes of resonant circuit is provided. Is reviewed previously unknown properties of the classical model of the resonant circuit. Apart from the fact that knowledge is never too much, can be developed new methods of measuring the parameters of electrical circuits, based on open source phenomena. It is also possible that an outdoor phenomenon has a physical explanation. Is a diagram of the mechanical model with similar properties.
\end{abstract}

Keywords: RCL Circuit, The Voltage Resonance, Special Point

\section{Introduction}

The article describes a previously unknown properties of an oscillatory contour, is inherent in both parallel and sequential type of circuits. The publication of these particulars may, as the authors suggest, to encourage attempts at physical explanation of the phenomenon open. It seems extremely important that the novelty of the information about the item, which is the basic component of countless electronic and electrical devices. In addition to proposing an expansion of the description of the circuit in the methodical and educational literature, we can expect the emergence of new methods of measurement and creating new measuring equipment. The authors have tried as faithfully as possible to explore the phenomenon inherent in any type of circuit. Since the interests of authors included and the analysis of electromechanical devices, the above analysis of the mechanical system, which describes the method used for the analysis of electric circuit. There is also the possibility that the described phenomenon is a mathematical curiosity, however, of interest for a better understanding of the processes occurring in the system, opened nearly two centuries earlier.

In textbooks and manuals on electrical engineering [1-4] is not carried out a full analysis of the properties of the oscillating circuit. Paradoxical properties of the model the parallel RLC circuit studied in [5-10] Frequency characteristics of the circuit contain the special point located at a frequency equal to $\frac{f_{r}}{\sqrt{2}}$, where the impedance does not depend on resistance. In these works investigated the existence region of a module of the impedance of the circuit and its phase characteristics. In the study of the imaginary components of the impedance detected another singular point, located at the resonant frequency. Valid component impedance has the shape close to the hyperboloid of rotation. Remains open the question of the physical interpretation of observed phenomena. In the present work we study the properties of a consistent model of RLC circuit, whose properties are studied in the textbooks of electrical engineering section - the resonant of voltage.

\section{Special Point for Serial Circuit}

Consider a model of sequential circuit constructed according to the scheme (Figure 1). This model is doubly impossible, since it contains an ideal inductance and an unrealistically bad (large leakage) capacitor. Apparently, 
because of these circumstances, this scheme has not been investigated previously.

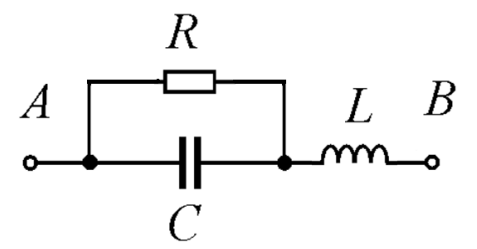

Figure 1. Electrical Circuit Model of the Circuit.

Impedance is easy to calculate by the formula:

$$
\bar{Z}=\frac{\left(1-\bar{\omega}^{2}\right) \bar{R}+j \bar{\omega}}{j \bar{\omega} \bar{R}+1}
$$

In (1) $\bar{Z}$ and $\bar{R}$ - complex and active resistance normalized by the characteristic resistance of the circuit is $\sqrt{\frac{L}{C}}$, and $\bar{\omega}$ is the frequency (circular) normalized by resonance frequency. The relative frequency equal to $\frac{1}{\sqrt{2}}$, the formula takes

$$
\bar{Z}=\frac{\frac{\bar{R}}{2}+\frac{j}{\sqrt{2}}}{j \frac{\bar{R}}{\sqrt{2}}+1}
$$

It is obvious that the module of relative impedance at this frequency does not depend on the resistance and equal to $\frac{1}{\sqrt{2}}$.

The vector phase of the semicircle at $\bar{R}=0$ equal $j \bar{\omega}$, and when $\bar{R} \rightarrow \infty$ equal $-j \frac{1-\bar{\omega}^{2}}{\bar{\omega}}$. Therefore, the radius of the semicircle is equal $\frac{1}{2 \bar{\omega}}$ and the offset of the center is equal $\frac{2 \bar{\omega}^{2}-1}{2 \bar{\omega}}$. When the relative frequency is $\frac{1}{\sqrt{2}}$, the displacement of the center is equal zero and the module does not depend on the magnitude of the relative resistance.

In Figure 2 presents the dependence of the current flowing in the circuit, the frequency at various values of relative resistance (at unity voltage).

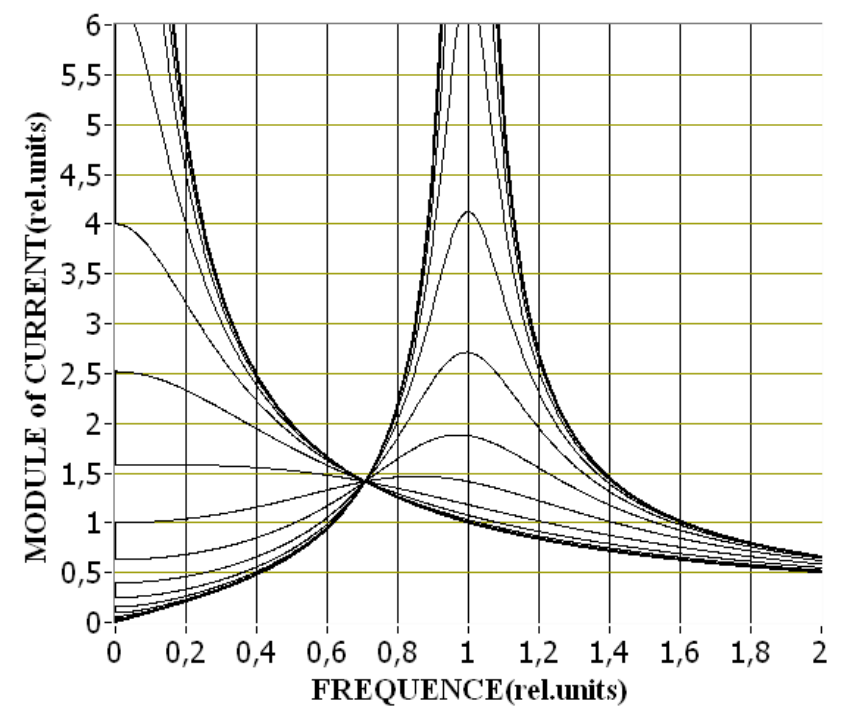

Figure 2. The Dependence of the Module of the Current in the Circuit Frequency.
In Figure 2 clear the area of existence of the current is bounded by two asymptotes $\frac{\left|1-\bar{\omega}^{2}\right|}{\bar{\omega}}$ when $\bar{R} \rightarrow \infty$ and $\frac{1}{\bar{\omega}}$ at $\bar{R}$ $=0$.

These characteristics coincide with the voltage on the parallel circuit (with a single current).

The current graph (figure 2) is given for convenience of comparison with the characteristics of the circuit, which are commonly used in textbooks on electrical engineering (section voltage resonance). In essence, it is the normalized conductivity of the chain under consideration. Calculations of a circuit with an additional active resistance are easier to conduct using the loop impedance. In further analysis it is based on the calculations of the impedance module.

Turning to a more realistic model of the circuit, sequentially connect the inductor and an additional resistance $\bar{r}$ (normalized by the characteristic resistance).

In Figure 3 the calculations of the impedance circuit when the magnitude of $\bar{r} \mathrm{r}=0.5$

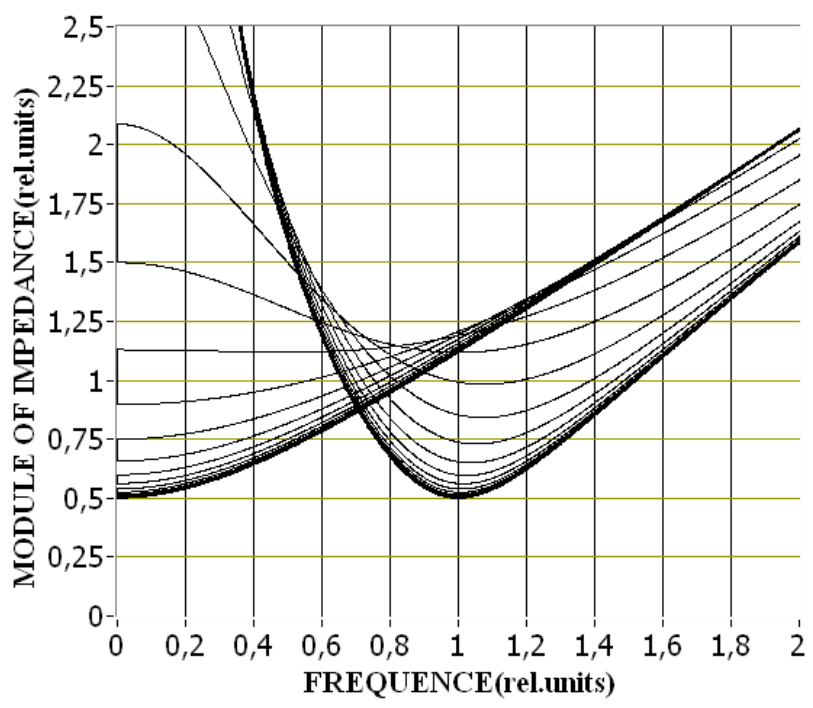

Figure 3. The Impedance of the Circuit When $\bar{r}=0.5$.

The region of existence of the impedance contour in the General case, limited to three asymtote:

1. Asymptote $\bar{Z}_{\text {min }}=\sqrt{\bar{r}^{2}+\bar{\omega}^{2}}$ for range of frequencies from 0 to $\frac{\bar{\omega}}{\sqrt{2}}$.

2. Asymptote $\bar{Z}_{\text {min }}=\sqrt{\bar{r}^{2}+\left(\frac{1-\bar{\omega}^{2}}{\bar{\omega}}\right)^{2}}$ for frequency $\frac{\bar{\omega}}{\sqrt{2}}$ to $\infty$.

3. Asymptote $\bar{Z}_{\max }=\sqrt{\bar{r}^{2}+\left(\bar{\omega}-\frac{1}{2 \bar{\omega}}\right)^{2}}+\frac{1}{2 \bar{\omega}}$

It is obvious that in this case there is a particular point on the frequency $\frac{\bar{\omega}}{\sqrt{2}}$. It is interesting to note that when $\bar{r} \rightarrow 0$ the third asymptote has two values of the derivative at the point $\frac{\bar{\omega}}{\sqrt{2}}$. In Figure 4 shows the dependence derived from the third asymptote to the frequency and resistance of inductors. It is obvious that the decrease in resistance there is a break curve and the neighborhood of a singular point. 


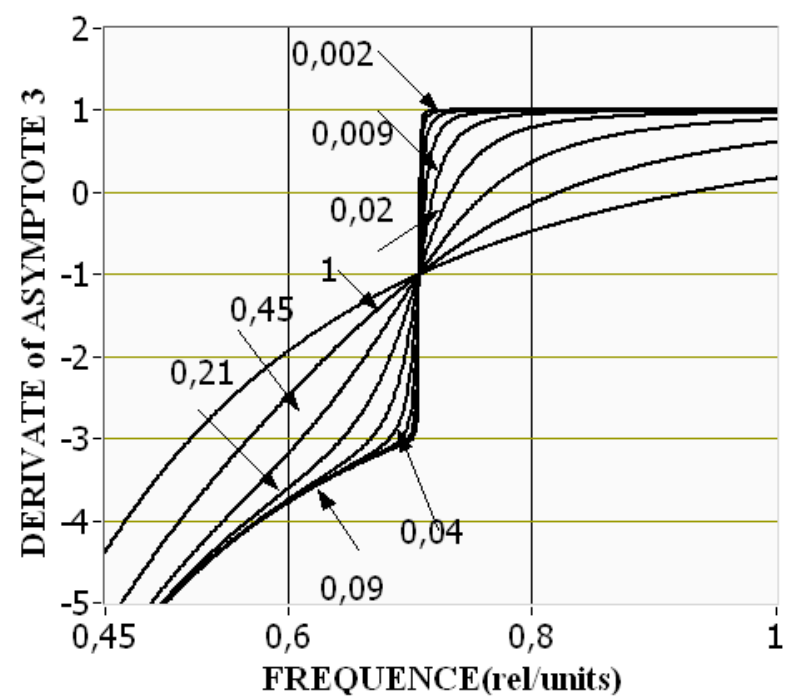

Figure 4. Derived from the Third Asymptote in Frequency Depending on the Active Resistance of the Inductor.

The local minimum of the third asymptote with increasing additional resistance increases from $\frac{\bar{\omega}}{\sqrt{2}}$ to infinity. When the value of $\bar{r}$ is equal to $\sqrt{2}$ is a local minimum at the resonant frequency. Frequency at least approaches the asymptote $\sqrt[3]{\frac{\bar{\omega}}{2}}$. In figure 5 asymptote is shown in broken lines.

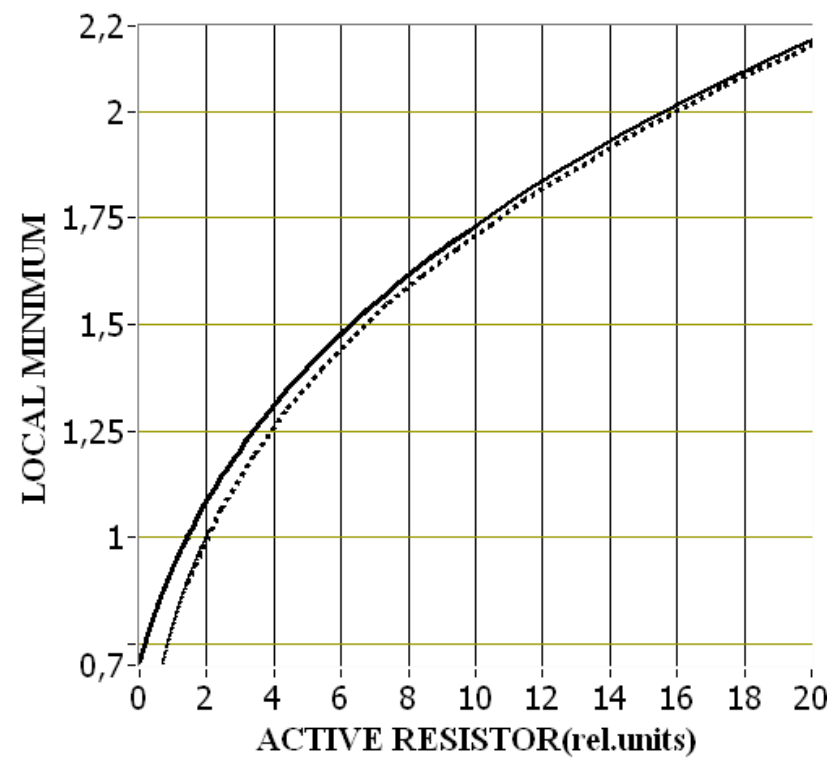

Figure 5. Local Minimum vs Value Relative Resistance.

The frequency of the local minimum of the third asymptote is determined by solving the equation (3)

$$
\bar{\omega}^{6}-0.75 \bar{\omega}^{2}+0.25\left(1-\bar{r}^{2}\right)=0
$$

\section{Comparison Serial and Parallel Circuit}

The findings can be applied to the parallel circuit constructed according to the scheme shown in Figure 4.

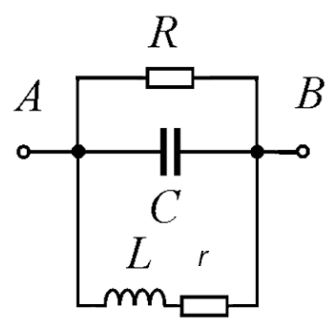

Figure 6. General Scheme for the Parallel Circuit.

The complex conductivity for a parallel circuit may be represented as:

$$
\bar{Y}=\frac{\left(1-\bar{\omega}^{2}\right) \overline{Y_{r}}+j \bar{\omega}}{j \bar{\omega} \overline{Y_{r}}+1}+\overline{Y_{R}}
$$

All of the conductivity normalized by the characteristic conductivity $\sqrt{\frac{C}{L}}$ in this formula.

It is easy to notice that the formula (4) coincides with (1) when connected to inductance of the additional resistance $-\bar{r}$. Consequently, all the results of the analysis of the circuit can be applied to the parallel circuit.

\section{Mechanic Model of Serial Circuit}

A mechanical analog of the considered type of the circuit shown in Figure 7.

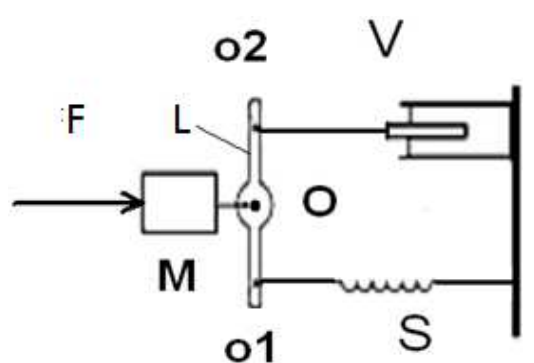

Figure 7. Scheme of the mechanical system with viscous friction.

$\mathrm{F}$ - the variable power, $\mathrm{S}$ - spring, - $\mathrm{O} 1$ - hinge spring, $\mathrm{L}$ - weightless lever, $\mathrm{O}$ - the main hinge, $\mathrm{O} 2$ - hinge of the second link, $\mathrm{M}$ - massive body, Velement viscous friction.

It is assumed that the elements of the system are located on a perfectly smooth a horizontal surface that allows you to exclude from the analysis the force of gravity. The calculations also assume relatively small mechanical displacement in which no change of the force direction.

The mechanical model consists of an upper subsystem, a massive body $M$ and the bottom containing spring and viscous element.

Weightless spring S (low mechanical losses), is characterized by the coefficient of flexibility $-\mathrm{C}$ is the inverse of stiffness.

The compliance of the subsystem of parallel electrical connection will be achieved when the equality of forces in both units (voltages) and of addition of velocities (currents). This goal is a weightless lever $\mathrm{L}$, which can rotate (without 
friction) around an axis $\mathrm{O}$ (Figure 7).

The weightlessness of the arm (its small mass compared to the mass of the second link) means that it can be applied to the following equation for the sum of the forces $\sum \mathrm{F}=0$ and moments $\sum \mathrm{M}=0$. In case of equal arms of the lever forces applied to the links will be equal to $F / 2$. The speed of movement of the axis of lever $V_{L}$ can be calculated as the sum of the speeds of the links

$$
V_{L}=\frac{V s+V v}{2}
$$

Vs - speed link with a spring, and the Vv - speed link with viscous element. In the result, the resonance frequency of the mechanical system is equal to

$$
\omega_{r}=\frac{2}{\sqrt{m C}}
$$

The total mechanical resistance of the system in complex form coincides with f.3. In this case the calculated resistance in the formula should be 4 times higher viscous resistance level of the lower subsystem.

\section{Conclusions}

It is shown that the singular point exists in the frequency characteristics of the serial RCL circuit. The region of existence of the impedance of the series resonant circuit is investigated. It is revealed that both types of contour are described by a single mathematical form of resistance in the serial circuit are changed to the conductivity in parallel. The model of the mechanical system is constructed, analogous to the serial circuit.

\section{References}

[1] MEASUREMENT AND CALIBRATION HANDBOOK Precision Measurement Equipment Laboratory... L. SERIES RCL EL, XL EA, ZT (Inductively). Circuit Analysis: Theory and Practice 5th Edition.

[2] Horowitz and Hill, the Art of Electronics, Second Edition, Cambridge University Press, 1989. ISBN 0521370957.

[3] US Navy, Basic Electrity, Dover, 1970. ISBN 0486209733.

[4] Http://www.wiley.com/college/comer/ ISBN 0471410160.

[5] Parfentev N. A. Paradoxes the Model of an RLC Circuit. Engineering Physics Volume 2, Issue 3, June 2017, Pages: 4953.

[6] Parfentyev N. A., Parfentyeva E. N., Sevastyanov S. I. Pecularity of frequency characteristics of a parallel oscillatory contour. News of higher education institutions. Electronics No. 62014, page 110.

[7] Parfentyev N. A. Invariant one model of a mechanical system with viscous friction, Electronic journal technical acoustics. 2014.

[8] Parfentyev N. A., Parfentyeva N. A., Truchanov S. V. On the singular point of the frequency characteristics of oscillatory systems. Natural and technical Sciences, №9-10, 2014, c. 31.

[9] Parfentyev N. A., Parfentyeva E. N., Krivolutsrfya N.V. Amazing properties of a parallel electrical circuit. XIX International Scientific and Practical Conference. Russia St. Petersburg, 19-20.02.2016 page.11-15.

[10] Parfentev N. A. New paraljxes the model of an RLC circuit. Science of Europe. N12. Vol. 1, page 19-23. 